\title{
Aspergilose em avestruz (Struthio camelus) no Brasil
}

\author{
Aspergillosis in an ostrich (Struthio camelus) in Brazil
}

\author{
Tatiane Alves da Paixão ${ }^{1}$ Ernane Fagundes do Nascimento ${ }^{2}$ \\ Paula Namie Shida Parra ${ }^{3}$ Renato de Lima Santos ${ }^{4}$
}

- RELATO DE CASO -

\section{RESUMO}

\begin{abstract}
Criatórios de avestruz têm se tornado comuns no Brasil, mas por se tratar de espécie exótica, as informações sobre as doenças desses animais no nosso ambiente são escassas. Um avestruz de cinco meses de idade apresentou tosse, anorexia, perda de peso, dispnéia discreta, temperatura corporal normal e morreu oito dias após o início dos sinais clínicos. Macroscopicamente foram observados nódulos múltiplos no pulmão e nos sacos aéreos. Histolopatogicamente, observou-se pneumonia granulomatosa e necrosante multifocal com hifas ramificadas e septadas intralesionais com características morfológicas de Aspergillus sp. Nos sacos aéreos, além das hifas e processo inflamatório adjacente haviam vários micélios com conidióforos na superfície interna. Foi isolado Aspergillus fumigatus dos tecidos afetados. De acordo com os achados histopatológicos e micológicos firmou-se o diagnóstico de pneumonia e aerossaculite micótica severa causada por Aspergillus fumigatus. Com base nas informações disponíveis na literatura, esse é o primeiro caso de aspergilose em avestruz documentado no Brasil.
\end{abstract}

Palavras-chave: avestruz, Aspergillus fumigatus, pneumonia, aerossaculite.

\section{ABSTRACT}

Farms raising ostriches are becoming popular in Brazil lately, but as an exotic species, there is little information regarding diseases that affect these animals in our environment. A five-month-old ostrich had cough, anorexia, weight loss, mild dispneia, normal body temperature, and died within 8 days from the beginning of the clinical signs. Grossly, there were multiple nodules in the lung and air sacs. Histopathologically, a multifocal necrotizing and granulomatous pneumonia with intralesional radiating septated and branching hyphae with morphological features of Aspergillus sp. was observed. In the air sacs, in addition to the hyphae and adjacent inflammatory reaction, there were multiple mycelia with conidiophores on the inner surface. Aspergillus fumigatus was isolated from the affected tissues. Based on the histopathological and mycological findings, a severe mycotic pneumonia and air sacculitis caused by Aspergillus fumigatus was diagnosed. To the best of our knowledge, this is the first reported case of aspergillosis in an ostrich in Brazil.

Key words: ostrich, Aspergillus fumigatus, pneumonia, air sacculitis.

\section{INTRODUÇÃO}

A criação comercial de avestruz (Struthio camelus) no Brasil tem se expandido muito recentemente. Como o avestruz é uma ave exótica e sua criação é uma atividade relativamente nova no país, pouco se sabe a respeito das doenças que acometem esses animais que possam ter importância econômica em nosso meio.

A aspergilose é uma das micoses sistêmicas mais comuns em aves, tendo sido previamente relatada em avestruzes na Austrália (ROUSSEAUX \& DALZIEL, 1981; KATZ et al., 1996), em Israel (PERELMAN \& KUTTIN, 1992a) e nos Estados Unidos (TERZICH \& VANHOOSER, 1993; MARKS et al., 1994). Contudo, até o presente, não existem casos

${ }^{1}$ Médico Veterinário, residente em Patologia Veterinária. Escola de Veterinária da Universidade Federal de Minas Gerais (UFMG), Belo Horizonte, MG.

${ }^{2}$ Professor adjunto, Doutor, Escola de Veterinária, UFMG

${ }^{3}$ Médico Veterinário, Avicultura Integral e Patologia Animal (AVIPA), Campinas, SP.

${ }^{4}$ Professor adjunto, PhD, Escola de Veterinária, UFMG, Departamento de Clínica e Cirurgia Veterinária, Av. Antônio Carlos, 6627, 30161-970 Belo Horizonte, MG. Fone: 31-3499-2239, Fax: 31-3499-2230. Email: rsantos@vet.ufmg.br. Autor para correspondência: 
de aspergilose relatados em avestruzes criados no Brasil. O presente relato descreve o diagnóstico de pneumonia e aeorossaculite por Aspergillus sp. em avestruz no Brasil.

\section{RELATO DO CASO}

Um avestruz de cinco meses de idade, proveniente de um criatório localizado no Estado de São Paulo, apresentou tosse, anorexia, perda de peso, dispnéia discreta, mas temperatura corporal normal. O curso clínico, entre o aparecimento dos sinais e a morte, foi de oito dias. $\mathrm{O}$ animal foi tratado com Enrofloxacina.

\section{RESULTADOS E DISCUSSÃO}

À necropsia, foram observados nódulos nos pulmões (de aproximadamente $0,5 \mathrm{~cm}$ de diâmetro) e nos sacos aéreos (variando de $1 \mathrm{~mm}$ a $5 \mathrm{~cm}$ de diâmetro). Fragmentos do pulmão e dos sacos aéreos foram processados de acordo com as técnicas rotineiras de inclusão em parafina, corados por HE, PAS e Grocott e examinados histologicamente. Microscopicamente, nos tecidos corados por HE, foram observados no parênquima pulmonar, múltiplos focos de infiltração histiocitária, em alguns casos associados com área central de necrose (Figura 1) contendo imagens negativas de hifas, circundadas por intenso infiltrado inflamatório constituído predominantemente por macrófagos (Figura 2). As hifas foram evidenciadas por colorações especiais como Grocott e PAS (Figura 1), que permitiram a observação de numerosas hifas de 6 a $10 \mu \mathrm{m}$ de comprimento, com paredes paralelas, septadas e regularmente ramificadas em ângulo agudo

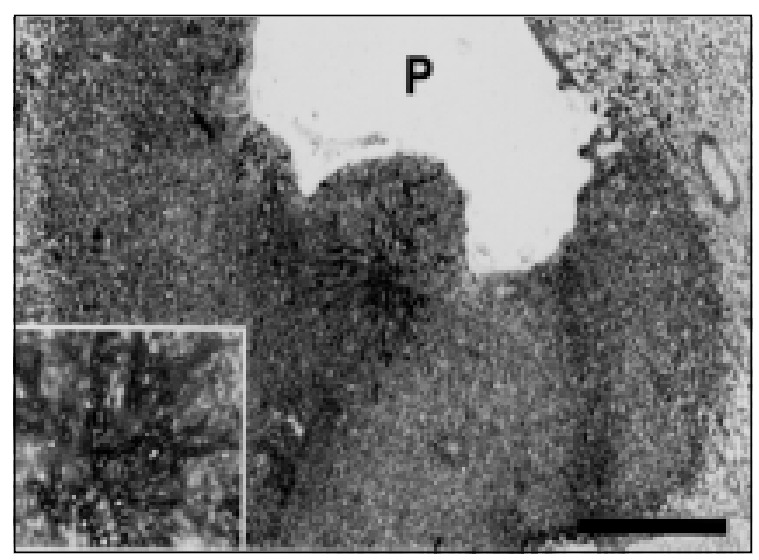

Figura 1 - Avestruz. Pulmão; foco granulomatoso adjacente a um parabrônquio (P), com hifas de Aspergillus sp. no centro da lesão (detalhe). PAS, barra $=300 \mu \mathrm{m}$.

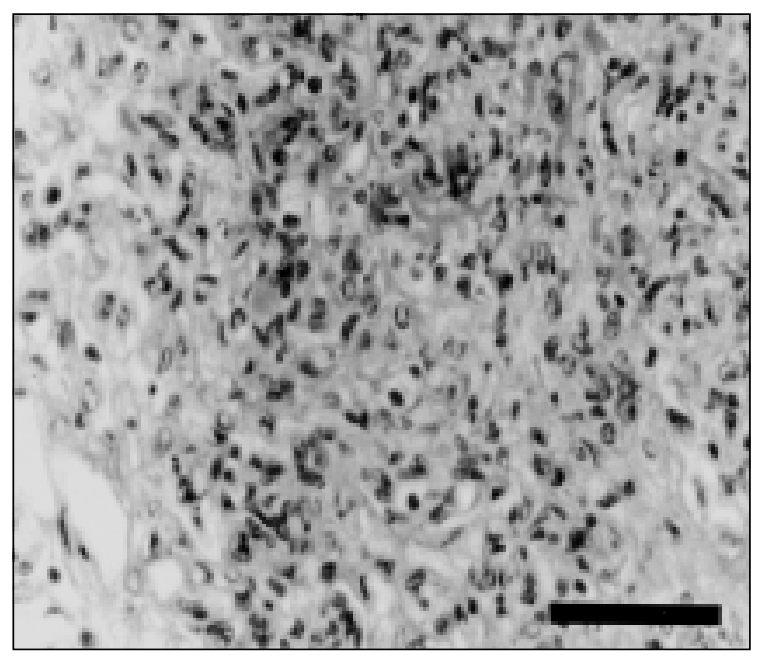

Figura 2 - Avestruz. Pulmão; infiltrado inflamatório focal predominantemente histiocitário. HE, barra $=37,5$ $\mu \mathrm{m}$.

compatível morfologicamente com hifas de Aspergillus sp. Em fragmentos da parede dos sacos aéreos, além da presença das hifas e reação inflamatória semelhantes àquelas do pulmão (Figura 3), foram observados, na superfície interna, micélios com conidióforos típicos de Aspergillus sp. (Figura 4). Cultivo em ágar Sabouraud de amostras das lesões do pulmão e dos sacos aéreos resultou no isolamento de Aspergillus fumigatus.

A zigomicose, infecção por fungos dos gêneros Rhizopus, Mucor e Basidia, foi considerada no diagnóstico diferencial. A zigomicose é uma micose

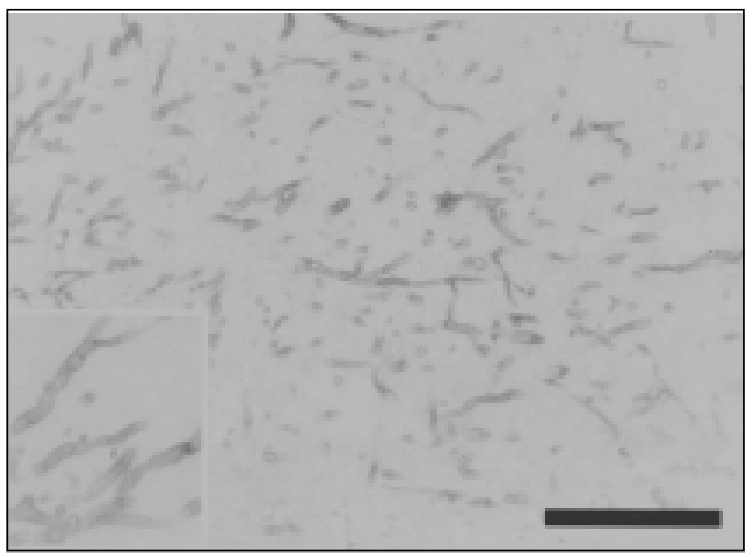

Figura 3 - Avestruz. Saco aéreo; superfície interna contendo inúmeras hifas de Aspergillus sp. Em detalhe, abaixo à direita, são observadas hifas septadas com ramificações em ângulo agudo. Grocott, barra $=75$ $\mu \mathrm{m}$.

Ciência Rural, v.34, n.2, mar-abr, 2004. 


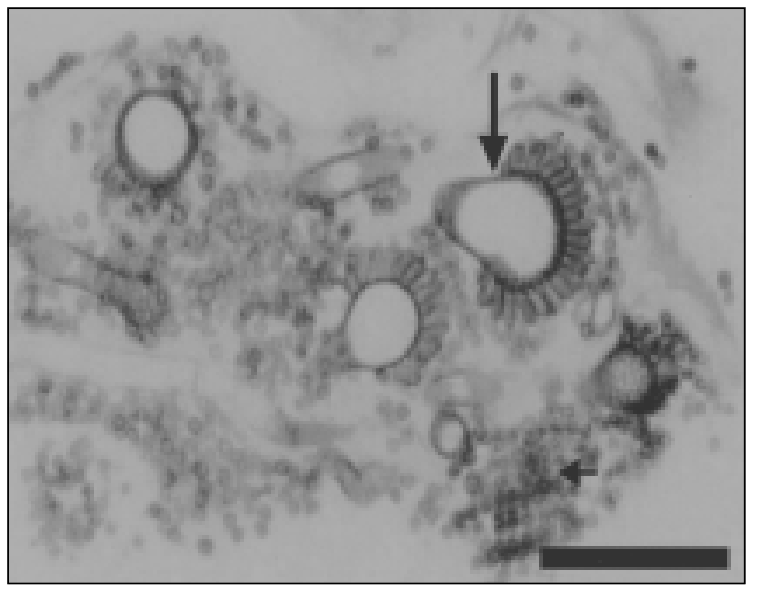

Figura 4 - Avestruz. Saco aéreo; superfície interna contendo vários micélios com conidióforos (seta grande) e esporos (seta pequena) de Aspergillus sp. Grocott, barra $=37,5 \mu \mathrm{m}$.

sistêmica menos comum do que a aspergilose em aves e que, geralmente, acomete o sistema digestivo (SHANE, 1998), embora possa estar também associada à doença respiratória (JEFFREY, et al., 1994). A zigomicose em avestruz tem sido associada a proventriculite e ventriculite com impactação (PERELMAN \& KUTTIN, 1992b; JEFFREY, et al., 1994; GULBAHAR et al., 2000). Além da distribuição das lesões, que são geralmente pulmonares no caso da aspergilose e predominantemente digestivas no caso da zigomicose, a morfologia dos agentes permite sua diferenciação. No caso da zigomicose as hifas têm de 7 a $20 \mu \mathrm{m}$ de comprimento, paredes irregulares, raramente são septadas e apresentam ramificações aleatórias. Em contraste, as hifas de Aspergillus sp. são septadas e se ramificam dicotomicamente em ângulos agudos. Além disso, as hifas de Aspergillus sp. nem sempre se coram pela hematoxilina e eosina (HE), enquanto as hifas dos zigomicetos são mais facilmente visualizadas em HE.

Aspergillus sp. é um fungo saprófita encontrado no ambiente, principalmente em material vegetal em decomposição. É a micose associada com doença respiratória mais freqüente em avestruzes. Geralmente está associada à pneumonia e aerossaculite granulomatosas. O agente etiológico mais freqüentemente envolvido é o Aspergillus fumigatus (SHANE, 1998), mas há relatos de pneumoniase aerossaculites em avestruzes causadas por A. niger e A. flavus (PERELMAN \& KUTTIN, 1992a). Os principais sinais clínicos são dispnéia, depressão e anorexia. A transmissão da aspergilose em avestruzes pode ser congênita, quando ocorre contaminação do ovo por esporos, ou adquirida após eclosão do ovo durante os primeiros dias de vida (SHANE, 1998). Em animais adultos, quando a infecção se estabelece por inalação de esporos está relacionada com condições de estresse, imunossupressão ou tratamento prolongado com antibióticos. A doença pode se manifestar por alta morbidade e alta mortalidade em aves jovens e, esporadicamente, acometer aves adultas (SHANE, 1998). Embora a infecção geralmente se manifeste como pneumonia e aerossaculite granulomatosas, há relato de um caso de rinite micótica causada por Aspergillus sp. em um avestruz (FITZGERALD \& MOISAN, 1995).

\section{CONCLUSÃO}

Com base nos achados histopatológicos e micológicos, firmou-se o diagnóstico de pneumonia e aerossaculite granulomatosas por Aspergillus fumigatus. De acordo com as informações bibliográficas disponíveis, esse parece ser o primeiro caso de aspergilose em avestruz documentado no Brasil.

\section{REFERÊNCIAS BIBLIOGRÁFICAS}

FITZGERALD, S.D.; MOISAN, P.G. Mycotic rhinitis in an ostrich. Avian Diseases, v.39, p.194-196, 1995.

GULBAHAR, M.Y.et al. Zygomycotid proventriculitis and ventriculitis in ostriches (Struthio camelus) with impaction. Australian Veterinary Journal, v.78, n.4, p.247-249, 2000 .

JEFFREY, J.S. Proventriculitis and ventriculitis associated with zygomycosis in ostrich chicks. Avian Diseases, v.38, p.630-634, 1994.

KATZ, M.E. Development of a method for the identification, using the polymerase chain reaction, of Aspergillus fumigatus isolated from ostriches. Australian Veterinary Journal, v.74, p.50-54, 1996.

MARKS, S.L.; STAUBER, E.H.; ERNSTROM, S.B Aspergillosis in an ostrich. Journal of the American Veterinary Medical Association, v.205, n.5, p.784-785, 1994.

PERELMAN, B.; KUTTIN, E.S. Aspergillosis in ostriches. Avian Pathology, v.21, p.159-163, 1992a.

PERELMAN, B.; KUTTIN, E. Zygomycosis in ostriches. Avian Pathology, v.21, p.675-680, 1992 b.

ROUSSEAUX, C.G.; DALZIEL, J.B. Aspergillus pneumonia in an ostrich (Struthio camelus). Australian Veterinary Journal, v.57, p.151-152, 1981. 
SHANE, S.M. Infectious diseases and parasites of ratites. Veterinary Clinics of North America: Food Animal Practice, v.14, n.3, p.455-483, 1998.
TERZICH, M.; VANHOOSER, S. Postmortem findings of ostriches submitted to the Oklahoma Animal Disease Diagnostic Laboratory. Avian Diseases, v. 37, p.1136-1141, 1993. 